5. Désogère $P$, Montesi SB, Caravan $P$. Molecular probes for imaging fibrosis and fibrogenesis. Chemistry 2019;25:1128-1141.

6. John AE, Luckett JC, Tatler AL, Awais RO, Desai A, Habgood A, et al. Preclinical SPECT/CT imaging of $\alpha \vee \beta 6$ integrins for molecular stratification of idiopathic pulmonary fibrosis. J Nucl Med 2013;54: 2146-2152.

7. Ambrosini V, Zompatori M, De Luca F, Antonia D, Allegri V, Nanni C, et al. 68Ga-DOTANOC PET/CT allows somatostatin receptor imaging in idiopathic pulmonary fibrosis: preliminary results. J Nucl Med 2010; 51:1950-1955.

8. Win T, Thomas BA, Lambrou T, Hutton BF, Screaton NJ, Porter JC, et al. Areas of normal pulmonary parenchyma on HRCT exhibit increased FDG PET signal in IPF patients. Eur J Nucl Med Mol Imaging 2014;41: 337-342.

9. Withana NP, Ma X, McGuire HM, Verdoes M, van der Linden WA, Ofori LO, et al. Non-invasive imaging of idiopathic pulmonary fibrosis using cathepsin protease probes. Sci Rep 2016;6:19755.

10. Win T, Screaton NJ, Porter JC, Ganeshan B, Maher TM, Fraioli F, et al. Pulmonary ${ }^{18} \mathrm{~F}-\mathrm{FDG}$ uptake helps refine current risk stratification in idiopathic pulmonary fibrosis (IPF). Eur J Nucl Med Mol Imaging 2018;45:806-815.

11. Wynn TA. Cellular and molecular mechanisms of fibrosis. J Pathol 2008;214:199-210.

Copyright (C) 2019 by the American Thoracic Society

\section{Digital Lung Auscultation: Will Early Diagnosis of Fibrotic Interstitial Lung Disease Become a Reality?}

\section{To the Editor:}

Interstitial lung diseases (ILDs) are a heterogeneous group of lung disorders that are often associated with substantial morbidity and early mortality. Pulmonary fibrosis is characteristic of most ILDs, and preliminary data suggest that early diagnosis and prompt intervention can improve clinical outcomes (1).

Idiopathic pulmonary fibrosis (IPF), one of the most common and best-studied forms of ILD, has a median estimated survival of 2-5 years from diagnosis (2). Delayed referral to a tertiary care center may be associated with higher mortality, irrespective of disease severity (3).

Early detection is essential to establish an accurate diagnosis and ensure timely intervention with appropriate treatment, as there are antifibrotics that have been approved for IPF (pirfenidone and nintedanib) and are effective in slowing disease progression (4) but do not reverse fibrosis. In addition, the decline in lung function for patients with IPF and preserved lung volume seems to be similar to that observed in those with more impaired lung volume ( $\sim 200 \mathrm{ml} / \mathrm{yr}$ ), and both groups appear to benefit equally from antifibrotic treatment (5).

Furthermore, patients with advanced IPF are more likely to discontinue treatment (6), adding to the rationale for earlier

Author Contributions: L.R., V.C., G.W., M.K., and G.S. contributed to the background literature search, providing appropriate references for inclusion. L.R., M.K., M.C., and G.S. suggested inclusion of the figure and provided input into how digital auscultation might fit into the algorithm for management of patients with idiopathic pulmonary fibrosis. All authors contributed to development of the manuscript in terms of critical review and amendment of initial drafted content, and approved the final submitted version.

Originally Published in Press as DOI: 10.1164/rccm.201902-0306LE on May 31, 2019 intervention. Here we discuss the potential role of digital lung auscultation as an aid to support the early identification of patients with suspected fibrotic ILD.

\section{Early Diagnosis of IPF Is Challenging}

Most patients experience significant challenges in their pursuit of a correct diagnosis of IPF (7). The nonspecific symptoms of IPF, including cough and insidiously progressive dyspnea, usually lead to repeated physician visits and multiple diagnostic tests, with misdiagnoses being commonplace (7).

Specific diagnostic procedures, such as chest high-resolution computed tomography (HRCT) to detect the patterns of usual interstitial pneumonia, lung biopsy, and multidisciplinary discussions, are recommended for an accurate diagnosis of IPF. Consideration of demographic and clinical data also improves diagnostic confidence (8).

Identification of interstitial lung abnormalities (ILAs), defined as the presence of specific patterns on chest computed tomography, may offer a strategy for early detection of IPF (9). The feasibility of this approach is dependent on an improved understanding of ILA subgroups and the nature of disease progression (9).

\section{Rationale for the Use of Digital Lung Auscultation}

Earlier access to a tertiary care center might be facilitated by improved methods of lung sound detection, including the recognition of so-called "Velcro-like" crackles, a hallmark feature that is present early in the disease course of IPF and in many fibrotic ILDs (10).

A subjective assessment of Velcro-like crackles bilaterally on chest auscultation predicts the presence of fibrotic ILD and usual interstitial pneumonia patterns on $\operatorname{HRCT}(10,11)$, and is associated with varying degrees of different ILAs (10). Hence, Velcro-like crackles may provide a rationale for investigating lung sounds to accurately identify patients with ILD, and could even be used as a screening aid. It has been suggested that the presence or absence of fine Velcro-like crackles at lung auscultation should be considered in the management of subjects with (sub)clinical ILD (12).

Methods for quantitative analysis of lung sounds have been shown to distinguish the Velcro-like crackles found in IPF from the lung sounds present in other pulmonary conditions that cause similar symptoms (13); research in this area is ongoing.

There is a clear need for further, clinically oriented, prospective studies to clarify the limitations and potential of the existing data. Previous studies have been affected by extensive variability in the methods used and features investigated; the complexity of the recording systems used (e.g., multichannel analyzers and aircoupled sensors); and a lack of robust diagnostic research designs for small, preselected study populations, usually with inappropriate reference to diagnostic standards.

\section{Steps Needed to Bring Digital Lung Auscultation into Clinical Practice}

Research projects are under way to build more evidence in this field. In an ongoing, multicenter clinical trial (clinicaltrials.gov identifier: NCT03503188), digital lung sounds and basic patient characteristics are being prospectively collected from patients with IPF and symptom-matched control subjects. A well-established teaching device for lung sounds (14) will be used as a reference. 


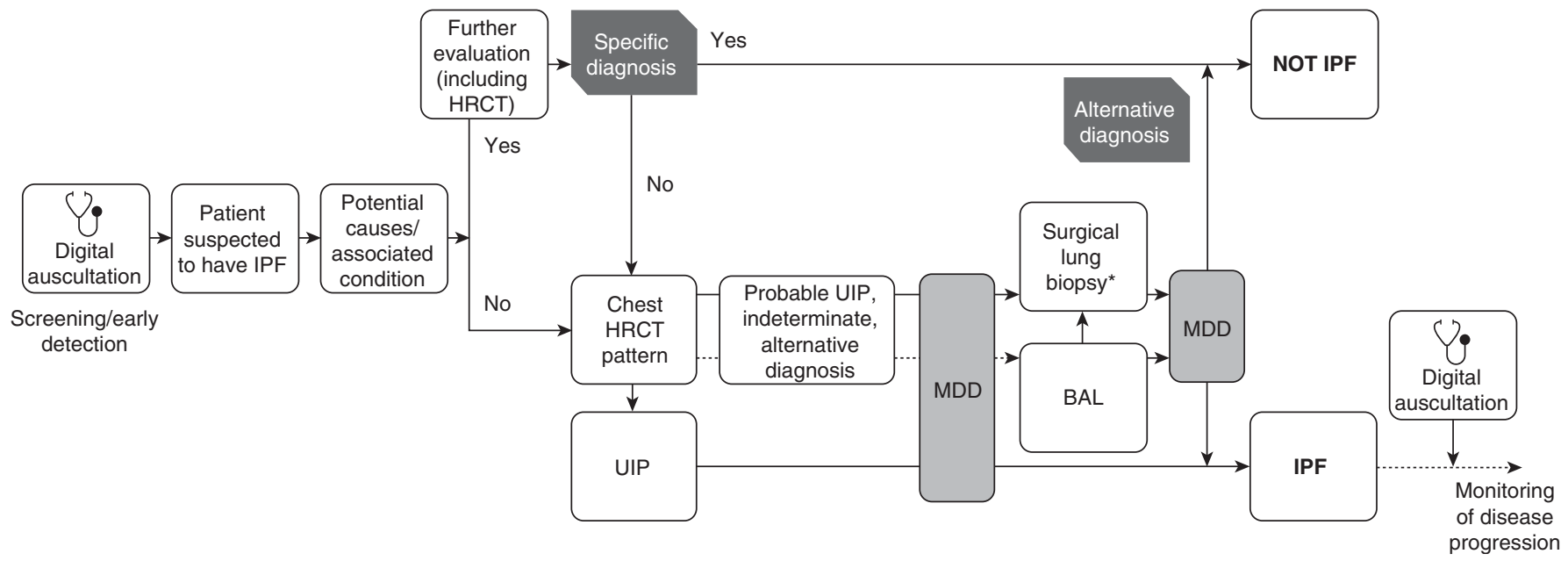

Figure 1. Potential role of digital lung auscultation in the management of patients with idiopathic pulmonary fibrosis. * Surgical lung biopsy is not suitable for all patients. HRCT = high-resolution computed tomography; IPF = idiopathic pulmonary fibrosis; MDD = multidisciplinary discussion; UIP = usual interstitial pneumonia. Reprinted from Reference 8.

The development and validation of supervised classification algorithms, using HRCT imaging as a reference, will assist the development of a digital auscultation aid to support the detection of specific acoustic patterns in IPF and facilitate early diagnosis (Figure 1). From a practical standpoint, we propose that such a tool should ultimately consist of a commercially available digital stethoscope (or alternatively a digital transductor connected to a standard stethoscope) and a software application to provide easy readouts of the probability of fibrotic lung disease. If validated, a digital device incorporating acoustic and readily available clinical data might provide an unprecedented opportunity to perform cost-effective, noninvasive screening of fibrotic ILD in at-risk populations. Indeed, we anticipate that the next greatest challenge will be to prove the effectiveness of such a tool in clinical practice, ideally requiring its implementation in primary care settings and a comparison with standard of care. The use of open-source platforms should also be strongly encouraged throughout the whole process to maximize refinement of the tool's diagnostic performance.

Digital lung sounds may have potential not only as a diagnostic aid but also as a quantitative methodology for monitoring disease progression in IPF (15). A correlation between lung sound changes and disease behavior has been shown in a proof-of-concept study (16), and further research is warranted.

\section{Conclusions}

By the time IPF is diagnosed, the patient's prognosis is often poor, and an opportunity for early treatment has been missed. As Velcrolike crackles on lung auscultation are present early in the disease course, digital assessment of patients' lung sounds, cross-referenced with a validated database of sounds from healthy lungs, different diseases, and other ILDs, may enable earlier and more accurate diagnosis and intervention. Artificial intelligence will hopefully aid in the development of accurate diagnostic algorithms based on digital auscultation data. Therefore, the development of a digital auscultation diagnostic aid has the potential to improve the care of patients with IPF and, conceivably, pulmonary fibrosis in general.

Author disclosures are available with the text of this letter at www.atsjournals.org.

Acknowledgment: The authors thank Lara Bennett, Ph.D., of AMICULUM Limited for providing medical writing support, funded by Boehringer Ingelheim.

Luca Richeldi, M.D.*

Fondazione Policlinico Universitario A. Gemelli IRCCS

Roma, Italy

Vincent Cottin, M.D.

University Claude Bernard Lyon 1

Lyon, France

Gebhard Würtemberger, M.D.

University of Freiburg

Freiburg, Germany

Michael Kreuter, M.D.

University of Heidelberg

Heidelberg, Germany

Mariarosaria Calvello, M.D.

Giacomo Sgalla M.D.

Fondazione Policlinico Universitario A. Gemelli IRCCS

Roma, Italy

ORCID IDs: 0000-0001-8594-1448 (L.R.); 0000-0003-3130-9388 (G.S.).

*Corresponding author (e-mail: luca.richeldi@policlinicogemelli.it).

\section{References}

1. Meyer KC. Diagnosis and management of interstitial lung disease. Trans/ Respir Med 2014;2:4. 
2. Molina-Molina M, Aburto M, Acosta O, Ancochea J, Rodríguez-Portal JA, Sauleda J, et al. Importance of early diagnosis and treatment in idiopathic pulmonary fibrosis. Expert Rev Respir Med 2018;12:537-539.

3. Lamas DJ, Kawut SM, Bagiella E, Philip N, Arcasoy SM, Lederer DJ. Delayed access and survival in idiopathic pulmonary fibrosis: a cohort study. Am J Respir Crit Care Med 2011;184:842-847.

4. Spagnolo $P$, Tzouvelekis A, Bonella $F$. The management of patients with idiopathic pulmonary fibrosis. Front Med (Lausanne) 2018;5:148.

5. Kolb M, Richeldi L, Behr J, Maher TM, Tang W, Stowasser S, et al. Nintedanib in patients with idiopathic pulmonary fibrosis and preserved lung volume. Thorax 2017;72:340-346.

6. Fletcher SV, Jones MG, Renzoni EA, Parfrey H, Hoyles RK, Spinks K, et al. Safety and tolerability of nintedanib for the treatment of idiopathic pulmonary fibrosis in routine UK clinical practice. ERJ Open Res 2018;4:00049-2018.

7. Schoenheit G, Becattelli I, Cohen AH. Living with idiopathic pulmonary fibrosis: an in-depth qualitative survey of European patients. Chron Respir Dis 2011;8:225-231.

8. Raghu G, Remy-Jardin M, Myers JL, Richeldi L, Ryerson CJ, Lederer DJ, et al.; American Thoracic Society, European Respiratory Society, Japanese Respiratory Society, and Latin American Thoracic Society. Diagnosis of idiopathic pulmonary fibrosis: an official ATS/ERS/ JRS/ALAT clinical practice guideline. Am J Respir Crit Care Med 2018; 198:e44-e68.

9. Wells AU, Kokosi MA. Subclinical interstitial lung abnormalities: toward the early detection of idiopathic pulmonary fibrosis? Am J Respir Crit Care Med 2016;194:1445-1446.

10. Sgalla G, Walsh SLF, Sverzellati N, Fletcher S, Cerri S, Dimitrov B, et al. "Velcro-type" crackles predict specific radiologic features of fibrotic interstitial lung disease. BMC Pulm Med 2018;18:103.

11. Sellarés J, Hernández-González F, Lucena CM, Paradela M, BritoZerón P, Prieto-González S, et al. Auscultation of Velcro crackles is associated with usual interstitial pneumonia. Medicine (Baltimore) 2016;95:e2573.

12. Cottin V, Cordier JF. Velcro crackles: the key for early diagnosis of idiopathic pulmonary fibrosis? Eur Respir J 2012;40:519-521.

13. Vyshedskiy A, Bezares F, Paciej R, Ebril M, Shane J, Murphy R. Transmission of crackles in patients with interstitial pulmonary fibrosis, congestive heart failure, and pneumonia. Chest 2005;128: 1468-1474.

14. Würtemberger G, Murphy Jun RLH. Auskultationskurs Lunge, AudioCD mit Begleitheft. Stuttgart, Germany: Thieme; 1997.

15. Key AL, Holt K, Warburton CJ, Walker PP, Earis JE. Use of zonal distribution of lung crackles during inspiration and expiration to assess disease severity in idiopathic pulmonary fibrosis. Postgrad Med J 2018;94:381-385.

16. Sgalla G, Larici AR, Sverzellati N, Bartholmai B, Walsh SLF, Nikolic D, et al. Quantitative analysis of lung sounds for monitoring idiopathic pulmonary fibrosis: a prospective pilot study. Eur Respir J 2018;53: 1802093

Copyright (C) 2019 by the American Thoracic Society

\section{Ә Fever in Sepsis: Still a Hot Topic}

To the Editor:

With great interest, we read the paper by Bhavani and colleagues (1), who report a novel method to distinguish between sepsis phenotypes based on body temperature. Using trajectory

əThis article is open access and distributed under the terms of the Creative Commons Attribution Non-Commercial No Derivatives License 4.0

(http://creativecommons.org/licenses/by-nc-nd/4.0/). For commercial usage and reprints, please contact Diane Gern (dgern@thoracic.org).

Originally Published in Press as DOI: 10.1164/rccm.201903-0484LE on March 25, 2019 modeling, the authors identify four phenotypes, each displaying distinct demographic characteristics, physiological parameters, and prognosis. The data presented reveal that the so-called hyperthermic, fast resolving patients, who presented with a high temperature that decreased rapidly afterward, had the lowest in-hospital mortality. In addition, a more swift increase in temperature was also positively related to survival. The authors plausibly argue that hyperthermic, fast resolvers may be able to mount a strong, but nevertheless wellbalanced, inflammatory response, resulting in a better clinical outcome.

What is currently missing is more solid evidence for whether or not there is an immunological basis for the proposed temperaturebased phenotypes. Insight into the release of so-called endogenous pyrogens (2), mainly represented by cytokines such as TNF $\alpha$ (tumor necrosis factor $\alpha$ ) and IL-6, could provide the missing link between the underlying immune status and the body temperature trajectories observed.

In the experimental human endotoxemia model, Escherichia coli endotoxin is administrated intravenously to healthy volunteers, inducing a standardized short-lasting systemic inflammatory response, which captures relevant hallmarks of the immune response observed in patients with sepsis (3). Serial measurements of cytokines and body temperature during endotoxemia allow for determination of their relationship. Using data from 20 male subjects allocated to the control group (no intervention other than bolus administration of $1 \mathrm{ng} / \mathrm{kg}$ endotoxin followed by continuous infusion of $1 \mathrm{ng} / \mathrm{kg} / \mathrm{h}$ endotoxin over the course of $3 \mathrm{~h}$ ) of a recent interventional study performed by our group (4), we analyzed the relationship between peak plasma cytokine concentrations and body temperature changes over time. We divided the subjects according to the median cytokine levels, resulting in 10 low and 10 high cytokine producers, and calculated the slope of the endotoxin-induced temperature increase $\left(\mathrm{Temp}_{\text {slope }}=\Delta\right.$ temperature increase/time to peak temperature).

On average, plasma concentrations of TNF $\alpha$, IL-6, and IL-10 reached their maximum at $2.5,3$, and 3.5 hours after endotoxin administration, respectively, whereas peak temperatures were observed 4 hours after endotoxin. Temp slope strongly correlated with peak temperature (Spearman $r=0.92 ; P<0.0001$ ). In subjects who produced high levels of the proinflammatory cytokines IL- 6 and TNF $\alpha$, Temp slope $_{\text {was significantly higher }}$ than in low proinflammatory cytokine producers, whereas Tempslope did not differ between low and high producers of antiinflammatory IL-10 (Figure 1). Spearman correlation coefficients for the relationship between cytokines and Temp slope $_{\text {were }} r=0.75$ for IL-6 $(P<0.0001)$ and $r=0.52$ for TNF $\alpha(P=0.02)$. Furthermore, peak temperatures were reached earlier by high IL- 6 and TNF $\alpha$ producers (high responders: $3.6 \pm 0.1$ vs. low responders: $4.5 \pm 0.2 \mathrm{~h}[P=0.002]$ and high responders: $3.7 \pm 0.2$ vs. low responders: $4.4 \pm 0.2 \mathrm{~h}$ $[P=0.02]$, respectively). After the peak, temperatures gradually normalized, but the slope of the temperature decrease did not correlate with levels of any of the measured cytokines.

Taken together, these findings indicate that indeed higher levels of proinflammatory, but not antiinflammatory, cytokines precede a more potent and swift temperature increase. These data provide 